\title{
HIERARCHICAL STRUCTURE FORMATION AND CHEMICAL EVOLUTION OF DAMPED Ly $\alpha$ SYSTEMS
}

\author{
Y.-Z. QIAN ${ }^{1}$ AND G. J. WASSERBURG ${ }^{2}$ \\ Received 2003 July 11; accepted 2003 August 19; published 2003 September 18
}

\begin{abstract}
We present a model for the chemical evolution of damped Ly $\alpha$ systems considering the production of "metals" by Type II supernovae and infall associated with hierarchical structure formation. The growth of metallicity in these systems is a reflection of the competition between astration and infall. The apparent late turn-on of these systems is due to the late cutoff of infall. The wide range in $[\mathrm{Fe} / \mathrm{H}]$ at a given redshift is explained by the range of the times for the onset of star formation and the range of the times for infall cessation in different systems. The observed lower bound of $[\mathrm{Fe} / \mathrm{H}] \approx-3$ follows from the very rapid initial rise of $[\mathrm{Fe} / \mathrm{H}]$ subsequent to the onset of star formation. To reach $[\mathrm{Fe} / \mathrm{H}] \approx-3$ from a metal-free initial state requires only $\sim 30 \mathrm{Myr}$, so the probability of observing lower $[\mathrm{Fe} / \mathrm{H}]$ values is very small.
\end{abstract}

Subject headings: galaxies: abundances — galaxies: evolution — intergalactic medium

\section{INTRODUCTION}

We discuss the chemical evolution of damped Ly $\alpha$ (DLA) systems on the basis of hierarchical structure formation. Observations (Lu et al. 1996; Prochaska \& Wolfe 2000, 2002; Prochaska et al. 2003) show that there is a baseline enrichment of $[\mathrm{Fe} / \mathrm{H}]=\log (\mathrm{Fe} / \mathrm{H})-\log (\mathrm{Fe} / \mathrm{H})_{\odot} \approx-3$ for DLA systems. Of 96 systems over the redshift range $0.5<z<5$, the lowest observed $[\mathrm{Fe} / \mathrm{H}]$ is -3.13 although the newer observations could have detected much lower values (Prochaska et al. 2003). Furthermore, there is a large dispersion in $[\mathrm{Fe} / \mathrm{H}]$ at any fixed $z$.

In an earlier discussion (Wasserburg \& Qian 2000b), we treated DLA systems as closed systems formed from the intergalactic medium (IGM) with a fixed initial inventory of "metals" (the prompt inventory) corresponding to $[\mathrm{Fe} / \mathrm{H}]_{P} \approx-3$ and assumed that the subsequent Fe enrichment of each system was governed by a constant $\mathrm{Fe}$ production rate per $\mathrm{H}$ atom in the gas of $P_{\mathrm{Fe}} /(\mathrm{H})$. Element production started when a system was formed at time $t^{*}$ after the big bang. At time $t>t^{*}$, the number ratio $(\mathrm{Fe} / \mathrm{H})$ of $\mathrm{Fe}$ atoms to $\mathrm{H}$ atoms in the gas evolves according to

$$
\frac{d(\mathrm{Fe} / \mathrm{H})}{d t}=\frac{P_{\mathrm{Fe}}}{(\mathrm{H})} .
$$

We only treat Fe contributions from Type II supernovae ( $\mathrm{SNe}$ II) in all our considerations as $\mathrm{SNe}$ Ia turn on at later times. Integration of equation (1) gives

$$
(\mathrm{Fe} / \mathrm{H})=(\mathrm{Fe} / \mathrm{H})_{P}+\frac{P_{\mathrm{Fe}}}{(\mathrm{H})}\left(t-t^{*}\right)
$$

As SNe II contributed $\sim(\mathrm{Fe} / \mathrm{H})_{\odot} / 3$ over the period of $(t-$ $\left.t^{*}\right) \sim 10$ Gyr prior to solar system formation, $P_{\mathrm{Fe}} /(\mathrm{H})$ is estimated to be $\sim(\mathrm{Fe} / \mathrm{H})_{\odot} /(30 \mathrm{Gyr})$.

In this model, the baseline $\mathrm{Fe}$ enrichment is explained by the prompt inventory and the dispersion in $[\mathrm{Fe} / \mathrm{H}]$ at a fixed $z$ by the range of $t^{*}$. It was found that most systems at any given $z$ have $t^{*}$ close to the age $t(z)$ of the universe at this $z$. This

\footnotetext{
School of Physics and Astronomy, University of Minnesota, Minneapolis, MN 55455; qian@physics.umn.edu.

${ }^{2}$ The Lunatic Asylum, Division of Geophysics and Planetary Sciences, California Institute of Technology, Pasadena, CA 91125.
}

implies that the "turn-on" of DLA systems occurs rather long after the big bang. Further, essentially all the data lie below the upper bound for $[\mathrm{Fe} / \mathrm{H}]$ corresponding to $t^{*}=0$. The above model appears to provide a reasonable description of the data on $[\mathrm{Fe} / \mathrm{H}]$ for DLA systems. This is rather remarkable considering that the model ignores infall. According to hierarchical structure formation, infall is essential to formation of DLA systems. Here we examine the chemical evolution of these systems by including infall. We wish to gain some insights into what causes most DLA systems at a given $z$ to have $t^{*}$ close to $t(z)$ in the closed-system model. We also address whether the prompt inventory is needed to explain the baseline Fe enrichment for DLA systems.

\section{CHEMICAL EVOLUTION WITH INFALL}

Consider a system of gas and stars with infall of primordial metal-free gas. The equations for evolution of the numbers of $\mathrm{Fe}$ and $\mathrm{H}$ atoms $[(\mathrm{Fe})$ and $(\mathrm{H})]$ in the gas are

$$
\begin{aligned}
\frac{d(\mathrm{Fe})}{d t} & =P_{\mathrm{Fe}}+(\mathrm{Fe} / \mathrm{H}) \frac{d(\mathrm{H})_{\mathrm{as}}}{d t}, \\
\frac{d(\mathrm{H})}{d t} & =\frac{d(\mathrm{H})_{\mathrm{as}}}{d t}+\frac{d(\mathrm{H})_{\mathrm{in}}}{d t},
\end{aligned}
$$

where $P_{\mathrm{Fe}}$ is the Fe production rate of SNe II, $d(\mathrm{H})_{\text {as }} / d t<0$ is the astration rate, and $d(\mathrm{H})_{\text {in }} / d t>0$ is the infall rate. When $\left|d(\mathrm{H})_{\text {as }} / d t\right|$ is small compared to $d(\mathrm{H})_{\mathrm{in}} / d t,(\mathrm{H})$ is governed by infall. Equations (3) and (4) give (Qian \& Wasserburg 2003)

$$
\frac{d(\mathrm{Fe} / \mathrm{H})}{d t}=\frac{P_{\mathrm{Fe}}}{(\mathrm{H})}-\frac{1}{(\mathrm{H})} \frac{d(\mathrm{H})_{\mathrm{in}}}{d t}(\mathrm{Fe} / \mathrm{H}) .
$$

Formal integration of equation (5) gives

$$
(\mathrm{Fe} / \mathrm{H})=\frac{P_{\mathrm{Fe}}}{(\mathrm{H})} t-\int_{0}^{t} \frac{1}{(\mathrm{H})} \frac{d(\mathrm{H})_{\mathrm{in}}}{d t^{\prime}}(\mathrm{Fe} / \mathrm{H}) d t^{\prime} .
$$

With the integral term interpreted as $P_{\mathrm{Fe}} t^{*} /(\mathrm{H})$, this resembles equation (2) for $(\mathrm{Fe} / \mathrm{H})_{P}=0$. So the effect of a sustained large infall rate is similar to late "turn-on."

In general, equations (4) and (5) must be solved together. 
For simplicity, we first consider the case where $d(\mathrm{H})_{\text {in }} / d t \gg$ $\left|d(\mathrm{H})_{\text {as }} / d t\right|$ so $(\mathrm{H}) \approx(\mathrm{H})_{\mathrm{in}}$. We then have

$$
\frac{d Z_{\mathrm{Fe}}}{d t}=\lambda_{\mathrm{Fe}}-\lambda_{\mathrm{in}} Z_{\mathrm{Fe}}
$$

where $Z_{\mathrm{Fe}} \equiv(\mathrm{Fe} / \mathrm{H}) /(\mathrm{Fe} / \mathrm{H})_{\odot}, \quad \lambda_{\mathrm{Fe}} \equiv P_{\mathrm{Fe}} /\left[(\mathrm{Fe} / \mathrm{H})_{\odot}(\mathrm{H})\right]$, and $\lambda_{\text {in }} \equiv d \ln (\mathrm{H})_{\text {in }} / d t$. If $d Z_{\mathrm{Fe}} / d t$ is negligible, a quasi-steady state is achieved and $Z_{\mathrm{Fe}}(t)$ approximately assumes the value $Z_{\mathrm{Fe}}^{\mathrm{QSS}}(t) \equiv \lambda_{\mathrm{Fe}}(t) / \lambda_{\mathrm{in}}(t)$. With the onset of Fe production at $t_{0}$, we have

$$
Z_{\mathrm{Fe}}(t) \approx Z_{\mathrm{Fe}}^{\mathrm{QSS}}(t)-Z_{\mathrm{Fe}}^{\mathrm{QSS}}\left(t_{0}\right) \exp \left[-\int_{t_{0}}^{t} \lambda_{\mathrm{in}}\left(t^{\prime}\right) d t^{\prime}\right]
$$

for $\lambda_{\text {in }}(t) \gg\left|d \ln Z_{\mathrm{Fe}}^{\mathrm{QSS}} / d t\right|$. At $t>t_{0}+\left[\lambda_{\text {in }}\left(t_{0}\right)\right]^{-1}$, the exponential term is negligible and $Z_{\mathrm{Fe}}(t) \approx Z_{\mathrm{Fe}}^{\mathrm{QSS}}(t)$.

We consider a baryonic system that is formed through infall into the potential well of a dark matter halo. The mass $M$ of the halo grows according to hierarchical structure formation. We assume that baryonic and dark matter are fed into the halo at a fixed mass ratio. The infall rate is then

$$
\lambda_{\mathrm{in}}(t)=\frac{d \ln M}{d t}=\frac{d \ln M}{d z} \frac{d z}{d t} .
$$

With $t \approx 17(1+z)^{-3 / 2}$ Gyr at $z>0.5$, equation (9) gives

$$
\lambda_{\mathrm{in}}(t) \approx \frac{4.4}{\mathrm{Gyr}}\left(\frac{\mathrm{Gyr}}{t}\right)^{5 / 3}\left|\frac{d \ln M}{d z}\right| .
$$

From Figure 6 in Barkana \& Loeb (2001), we find that $|d \ln M / d z| \sim 2.3$ (within a factor of 2) for $0.5<z<5$. As an example, we take $\lambda_{\text {in }}(t)=(0.1 \mathrm{Gyr})^{-1}(\mathrm{Gyr} / t)^{5 / 3}$ and $\lambda_{\mathrm{Fe}}=$ $(30 \mathrm{Gyr})^{-1}$ for $t>0$ (i.e., $t_{0}=0$ ). We numerically integrate equation (7) and show the evolution of $[\mathrm{Fe} / \mathrm{H}]=\log Z_{\mathrm{Fe}}$ in Figure 1. The quasi-steady state value $Z_{\mathrm{Fe}}^{\mathrm{OSS}}(t)=$ $\lambda_{\mathrm{Fe}} / \lambda_{\mathrm{in}}(t)=(t / \mathrm{Gyr})^{5 / 3} / 300$ is a good approximation to the exact solution. For the case of evolution from a metal-free initial state without infall, $Z_{\mathrm{Fe}}(t)=\lambda_{\mathrm{Fe}} t$ (the case of $t^{*}=0$ in $\S 1$ ). In comparison with this, Fe enrichment in the case of infall is suppressed by a factor of $\approx \lambda_{\text {in }}(t) t \approx 10(\mathrm{Gyr} / t)^{2 / 3}$, which ranges from 4.5 for $t=3.3 \mathrm{Gyr}(z=2)$ to 8.9 for $t=1.2 \mathrm{Gyr}$ $(z=5)$. This would explain the late "turn-on" requiring $t^{*}$ to be close to $t(z)$ in the closed-system model. The data on [Fe/ H] for 96 DLA systems (Prochaska et al. 2003) are shown in Figure 1. It can be seen that the solid curve for the case of infall passes through the median of the body of the data. This curve has a slope of $d[\mathrm{Fe} / \mathrm{H}] / d z \approx-1.1 /(1+z)$. For $z \gtrsim 2$ where most of the data lie, this slope is in good agreement with the estimate of $d[\mathrm{Fe} / \mathrm{H}] / d z \approx-0.26$ given by Prochaska et al. (2003) for the growth of the mean $[\mathrm{Fe} / \mathrm{H}]$ as a function of $z$. However, the above result does not explain the wide range in $[\mathrm{Fe} / \mathrm{H}]$ at a given $z$.

\section{THE DISPERSION IN $[\mathrm{Fe} / \mathrm{H}]$}

We now turn to the wide range in $[\mathrm{Fe} / \mathrm{H}]$ for DLA systems at a given $z$. We assume a constant Fe production rate $\lambda_{\mathrm{Fe}}$ per $\mathrm{H}$ atom in the gas for $t>t_{0}$. The infall rate $\lambda_{\text {in }}$ is estimated on the basis of hierarchical structure formation, and we now allow the possibility that infall may cease at a time $t_{\mathrm{IC}}$. We assume

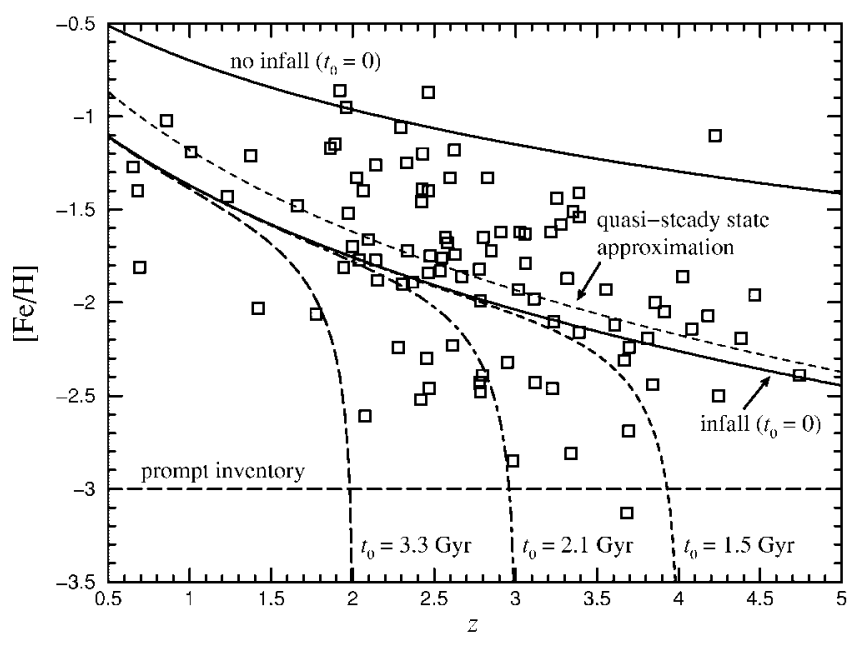

FIG. 1.-Data (squares) on $[\mathrm{Fe} / \mathrm{H}]$ for 96 DLA systems (Prochaska et al. 2003). The solid curve labeled "infall" shows the evolution of $[\mathrm{Fe} / \mathrm{H}]$ for a system with infall and a constant Fe production rate $\lambda_{\mathrm{Fe}}$ per $\mathrm{H}$ atom in the gas since the big bang. The thin dashed curve is the quasi-steady state approximation. The solid curve labeled "no infall" is for a system with a constant $\lambda_{\mathrm{Fe}}$ since the big bang but with no infall. The short-dashed curve is for a system in which infall starts at the big bang but star formation starts at $t_{0}=1.5 \mathrm{Gyr}\left(z_{0}=4\right)$, the dot-dashed curve is for $t_{0}=2.1 \mathrm{Gyr}\left(z_{0}=3\right)$, and the long-dashed curve is for $t_{0}=3.3 \mathrm{Gyr}\left(z_{0}=2\right)$. Assuming a halo mass of $M_{0}=5 \times 10^{8} M_{\odot}$ at the onset of star formation, $z_{0}=4,3$, and 2 correspond to $1.1 \sigma, 0.9 \sigma$, and $0.7 \sigma$ density fluctuations, respectively. Because of the very rapid initial rise of $[\mathrm{Fe} / \mathrm{H}]$, the probability of sampling DLA systems with $[\mathrm{Fe} / \mathrm{H}]<-3$ is very small. The prompt inventory proposed earlier (horizontal dashed line) is shown for reference.

that the infall rate greatly exceeds the astration rate at $t<t_{\mathrm{IC}}$. Under these assumptions, $t_{0}$ and $t_{\mathrm{IC}}$ determine $[\mathrm{Fe} / \mathrm{H}]$ in a baryonic system at time $t$ (see eqs. [8] and [11]).

First consider the case where $t_{0}=0$ and infall ceases at $t_{\mathrm{IC}}$. The evolution of $Z_{\mathrm{Fe}}$ is the same as for the case of infall discussed in $\S 2$ until $t=t_{\mathrm{IC}}$. At $t>t_{\mathrm{IC}}$, equation (7) reduces to $d Z_{\mathrm{Fe}} / d t=\lambda_{\mathrm{Fe}}$, which gives

$$
Z_{\mathrm{Fe}}(t)=Z_{\mathrm{Fe}}\left(t_{\mathrm{IC}}\right)+\lambda_{\mathrm{Fe}}\left(t-t_{\mathrm{IC}}\right) .
$$

Solutions for $t_{\mathrm{IC}}=0,1.5 \mathrm{Gyr}\left(z_{\mathrm{IC}}=4\right)$, and $4.3 \mathrm{Gyr}\left(z_{\mathrm{IC}}=\right.$ 1.5) are shown in Figure 2. In all cases of $t_{\mathrm{IC}}>0$, the evolution of $[\mathrm{Fe} / \mathrm{H}]$ for $t<t_{\mathrm{IC}}$ is along the solid curve labeled "continuous infall." As illustrated by points A, B, and C at $z=3$ in Figure 2 , a wide range of $[\mathrm{Fe} / \mathrm{H}]$ bounded by the cases of no infall $\left(t_{\mathrm{IC}}=0\right)$ and continuous infall can be produced at a given $z$ for different $t_{\mathrm{IC}}$ values.

Next consider the case where infall is continuous but $t_{0}$ may vary. Subsequent to onset of $\mathrm{Fe}$ production at $t_{0}, Z_{\mathrm{Fe}}$ grows rapidly and approaches $Z_{\mathrm{Fe}}^{\text {OSS }}$ on a timescale of $\sim \lambda_{\text {in }}\left(t_{0}\right)^{-1}$ (see eq. [8]). This is shown in Figure 1 for $t_{0}=1.5 \mathrm{Gyr}\left(z_{0}=4\right)$, $2.1 \mathrm{Gyr}\left(z_{0}=3\right)$, and $3.3 \mathrm{Gyr}\left(z_{0}=2\right)$. For example, $[\mathrm{Fe} / \mathrm{H}]$ in baryonic systems formed at $t_{0}=2.1 \mathrm{Gyr}$ first evolves rapidly, crossing $[\mathrm{Fe} / \mathrm{H}]=-3$, and then grows more slowly toward the quasi-steady state solution. Thus, values of $[\mathrm{Fe} / \mathrm{H}]$ below those for the case of $t_{0}=0$ may be populated with baryonic systems that have different $t_{0}$ values. Then the lower range in $[\mathrm{Fe} / \mathrm{H}]$ at a given $z$ can also be explained by the infall model.

The observed lower bound of $[\mathrm{Fe} / \mathrm{H}] \approx-3$ for DLA systems requires attention. With infall, the growth of $Z_{\mathrm{Fe}}$ is determined by competition between $\lambda_{\mathrm{Fe}}$ and $\lambda_{\text {in }} Z_{\mathrm{Fe}}$. As $Z_{\mathrm{Fe}}\left(t_{0}\right)=0, \lambda_{\mathrm{Fe}}$ governs the initial growth of $Z_{\mathrm{Fe}}$ so long as $\lambda_{\mathrm{Fe}} / \lambda_{\mathrm{in}} \gg Z_{\mathrm{Fe}}$. We 


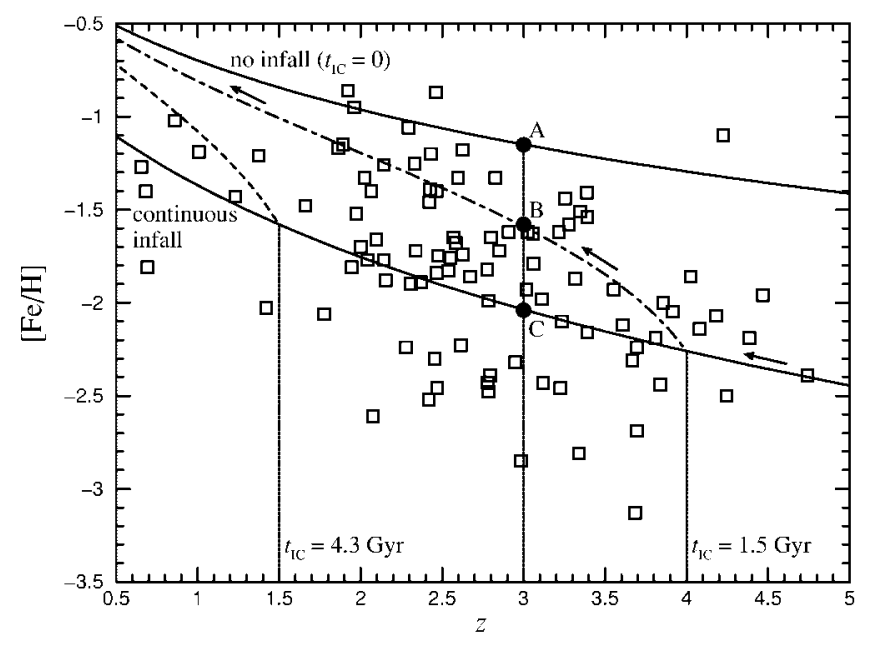

FIG. 2.-Data and the two solid curves are the same as in Fig. 1. The dotdashed curve shows the evolution of $[\mathrm{Fe} / \mathrm{H}]$ at $t>t_{\mathrm{IC}}$ for a system in which infall starts at the big bang but ceases at $t_{\mathrm{IC}}=1.5 \mathrm{Gyr}\left(z_{\mathrm{IC}}=4\right)$, and the dashed curve is for $t_{\mathrm{IC}}=4.3 \mathrm{Gyr}\left(z_{\mathrm{IC}}=1.5\right)$. The overall evolution of $[\mathrm{Fe} /$ $\mathrm{H}]$ for the system with $t_{\mathrm{IC}}=1.5 \mathrm{Gyr}$ is indicated by the arrows. In all cases, star formation starts at the big bang $\left(t_{0}=0\right)$. Points $\mathrm{A}, \mathrm{B}$, and $\mathrm{C}$ at $z=3$ represent $[\mathrm{Fe} / \mathrm{H}]$ values sampled by DLA systems with different $t_{\mathrm{IC}}$ values. Assuming a halo mass of $M_{\mathrm{IC}}=10^{11} M_{\odot}\left(\approx 10^{10} M_{\odot}\right.$ in baryonic matter $)$ at the time of infall cessation, $z_{\mathrm{IC}}=4$ and 1.5 correspond to $1.9 \sigma$ and $0.95 \sigma$ density fluctuations, respectively.

note that $\lambda_{\mathrm{Fe}} / \lambda_{\text {in }}=(t / \mathrm{Gyr})^{5 / 3} / 300 \gg 10^{-3}$ for $t \gg 0.49 \mathrm{Gyr}$ $(z \ll 9.6)$. So for systems with $t_{0}>1.2 \mathrm{Gyr}\left(z_{0}<5\right)$, the growth of $Z_{\mathrm{Fe}}$ up to $10^{-3}$ is essentially governed by $\lambda_{\mathrm{Fe}}=(30$ Gyr $)^{-1}$. These systems achieve $Z_{\mathrm{Fe}}=10^{-3}$ in $\sim 30 \mathrm{Myr}$ and then approach $Z_{\mathrm{Fe}} \approx Z_{\mathrm{Fe}}^{\mathrm{QSS}}>10^{-3}$ on a timescale of $\sim \lambda_{\text {in }}\left(t_{0}\right)^{-1}$ (see eq. [8] and Fig. 1). Thus, all baryonic systems will reach $Z_{\mathrm{Fe}}=10^{-3}$ or greater in a very short time at $z<5$, and the probability of observing DLA systems with $[\mathrm{Fe} / \mathrm{H}]<-3$ is quite small.

The ranges of $t_{0}$ and $t_{\mathrm{IC}}$ in the infall model to explain the dispersion in $[\mathrm{Fe} / \mathrm{H}]$ at a given $z$ are analogous to the range of $t^{*}$ in the closed-system model. However, uniform growth of $[\mathrm{Fe} / \mathrm{H}]$ occurs only at $t>t_{\mathrm{IC}}$ in the infall model. It is the dilution of gas by fresh infalling baryonic material that retards $[\mathrm{Fe} / \mathrm{H}]$ from uniform growth although Fe production and astration have been going on since $t_{0}$. To relate $t_{0}$ and $t_{\mathrm{IC}}$ for a baryonic system to the properties of the halo hosting the system, we follow the discussion in Barkana \& Loeb (2001). The condition for a halo of mass $M$ associated with an $n \sigma$ density fluctuation (an $n \sigma$ halo) to collapse at redshift $z$ is

$$
1.33(1+z) \approx n \sigma(M)
$$

where $\sigma(M)$ is shown in Figure 5 of Barkana \& Loeb (2001). Equation (12) gives the $z$ values at which a fixed mass $M$ is reached by halos with different $n$ values.

A $1 \sigma$ halo today has $M \approx 10^{13} M_{\odot}$ and should contain $\approx 10^{12}$ $M_{\odot}$ of baryonic matter. This mass is far greater than found for a typical galaxy. Thus, we may consider that infall into individual protogalaxies ceases when the accreted baryonic matter reaches $\sim 10^{10}-10^{11} M_{\odot}$ in a halo of $\sim 10^{11}-10^{12} M_{\odot}$. In the hierarchical model, a halo of larger masses (e.g., $\gtrsim 10^{12} M_{\odot}$ ) may consist of a number of smaller collapsed halos instead of a single object. This also applies to the associated baryonic systems. We assume that infall ceases at $t_{\mathrm{IC}}$ when a halo reaches a mass of $M_{\mathrm{IC}}=10^{11} M_{\odot}\left[\sigma\left(M_{\mathrm{IC}}\right)=3.5\right]$. This occurs at redshifts $z_{\mathrm{IC}}=4$ and 1.5 for $1.9 \sigma$ and $0.95 \sigma$ halos, respectively.
These redshifts correspond to the $t_{\mathrm{IC}}$ in Figure 2 and seem reasonable. For $n \sigma$ halos with $n>4, M_{\mathrm{IC}}=10^{11} M_{\odot}$ is reached at $z_{\mathrm{IC}}>9.5\left(t_{\mathrm{IC}}<0.5 \mathrm{Gyr}\right)$. The evolution of $[\mathrm{Fe} / \mathrm{H}]$ at $z<5$ $(t>1.2 \mathrm{Gyr})$ for baryonic systems inside these high- $\sigma$ halos is close to the case of $t_{\mathrm{IC}}=0$. Similar results are found for $M_{\mathrm{IC}} \sim 10^{11}-10^{12} M_{\odot}$.

Now consider $t_{0}$, which may be taken as the onset of star formation. Following the discussion in Barkana \& Loeb (2001), the first stars appear to have formed in $\sim 3 \sigma-4 \sigma$ halos of mass $M \sim 10^{5} M_{\odot}[\sigma(M) \approx 10]$ at $z \sim 20-30$. For these halos, $t_{0}$ is only $\sim 0.1$ Gyr. Formation of stars at $z<20$ appears to require a minimum halo mass of $\sim 10^{8}-10^{9} M_{\odot}$. We assume that star formation starts at $t_{0}$ when a halo reaches a mass of $M_{0}=$ $5 \times 10^{8} M_{\odot}\left[\sigma\left(M_{0}\right)=6\right]$. This occurs at redshifts $z_{0}=4,3$, and 2 for $1.1 \sigma, 0.89 \sigma$, and $0.67 \sigma$ halos, respectively. These redshifts correspond to the $t_{0}$ in Figure 1 and again seem reasonable. Similar results are found for $M_{0} \sim 10^{8}-10^{9} M_{\odot}$.

It follows that at a given $z$, baryonic systems inside different halos are in different stages of evolution. Consider an example using $M_{0}=5 \times 10^{8} M_{\odot}$ and $M_{\mathrm{IC}}=10^{11} M_{\odot}$. At $z=2.6$, star formation just starts in a $0.8 \sigma$ halo $([\mathrm{Fe} / \mathrm{H}] \sim-3)$, Fe production competes with infall in a $1.1 \sigma$ halo $([\mathrm{Fe} / \mathrm{H}]=-1.9$; see Fig. 1), and uniform growth of $[\mathrm{Fe} / \mathrm{H}]$ has been going on since infall cessation at $z_{\mathrm{IC}}=4$ in a $1.9 \sigma$ halo $([\mathrm{Fe} / \mathrm{H}]=-$ 1.4; see Fig. 2). For a sample of DLA systems at $z=2.6$, systems with $-3<[\mathrm{Fe} / \mathrm{H}] \leq-1.9,-1.9<[\mathrm{Fe} / \mathrm{H}] \leq-1.4$, and $[\mathrm{Fe} / \mathrm{H}]>-1.4$ are then associated with $n \sigma$ halos with $0.8<$ $n \leq 1.1,1.1<n \leq 1.9$, and $n>1.9$, respectively. Statistically speaking, the fraction of halos more evolved than an $n \sigma$ halo is $F(n)=\sqrt{2 / \pi} \int_{n}^{\infty} \exp \left(-x^{2} / 2\right) d x$. Thus, we expect the occurrences of $-3<[\mathrm{Fe} / \mathrm{H}] \leq-1.9,-1.9<[\mathrm{Fe} / \mathrm{H}] \leq-1.4$, and $[\mathrm{Fe} / \mathrm{H}]>-1.4$ at $z=2.6$ to be in the ratios of $[F(0.8)-$ $F(1.1)]:[F(1.1)-F(1.9)]: F(1.9) \approx 0.7: 1: 0.3$. Of 19 DLA systems at $2.4<z<2.7$, the numbers of systems in the above $[\mathrm{Fe} / \mathrm{H}]$ intervals are 5,10 , and 4 , consistent with expectation from our model.

\section{DISCUSSION AND CONCLUSIONS}

We have treated the chemical evolution of DLA systems considering astration and infall. It is assumed that the $\mathrm{H}$ in the gas is initially controlled by the infall of primordial metal-free baryonic matter and the $\mathrm{Fe}$ production rate per $\mathrm{H}$ atom in the gas is constant subsequent to the onset of star formation in a system. With the infall rate estimated from the standard scenario of hierarchical structure formation, this model yields an explanation for the data on $[\mathrm{Fe} / \mathrm{H}]$ in DLA systems. It is shown that the slow growth of $[\mathrm{Fe} / \mathrm{H}]$ with decreasing $z$ is a direct consequence of competition between enrichment by Fe production and dilution by infall. It is argued that the upper range of $[\mathrm{Fe} / \mathrm{H}]$ at a given $z$ results from the different times at which individual halos associated with protogalaxies reach a mass of $\sim 10^{11} M_{\odot}$ and infall ceases or greatly diminishes. It is also argued that the lower range of $[\mathrm{Fe} / \mathrm{H}]$ results from the different times at which individual halos reach a minimum mass of $\sim 10^{8}$ $10^{9} M_{\odot}$ required to initiate astration. Because the initial growth of $[\mathrm{Fe} / \mathrm{H}]$ up to -3 is very rapid subsequent to onset of astration, the probability of observing DLA systems with $[\mathrm{Fe} / \mathrm{H}]<-3$ is very small. The approach presented here appears to be a reasonably quantitative description of the chemical evolution of DLA systems that is compatible with the paradigm of hierarchical structure formation. Of the available data, only three points lie outside the upper bound of the model, and these are still within a factor of 2 of this bound. 
We assumed that the astration rate is small compared to the infall rate during the infall phase. If we assume a constant astration rate $\alpha$ per $\mathrm{H}$ atom in the gas consistent with the $\mathrm{Fe}$ production rate, then equation (4) reduces to $d(\mathrm{H}) / d t=-\alpha(\mathrm{H})+d(\mathrm{H})_{\text {in }} / d t$, which can be solved explicitly. As long as $d(\mathrm{H})_{\text {in }} / d t>\alpha(\mathrm{H}),(\mathrm{H})$ increases. When infall ceases, $(\mathrm{H})$ reaches the maximum value $(\mathrm{H})_{\max }$ and the star formation rate also reaches the maximum value $\alpha(\mathrm{H})_{\max }$. Subsequently, the gas will be depleted by star formation on a timescale of $\sim \alpha^{-1}$. When a protogalaxy would reach its maximum star formation rate depends on when its associated halo reaches a mass of $\sim 10^{11} M_{\odot}$ and infall ceases. This occurs at $z \sim 1.6$ and 4.3 for $1 \sigma$ and $2 \sigma$ halos, respectively. The extent to which the approach outlined here can be used to quantitatively explain the cosmic star formation history remains to be explored.

In earlier works (Wasserburg \& Qian 2000a; Qian \& Wasserburg 2002), we proposed that very massive $\left(\gtrsim 100 M_{\odot}\right)$ stars (VMSs) produced the elements from $\mathrm{C}$ to the $\mathrm{Fe}$ group to explain the observed jump in the abundances of heavy $r$-process elements (Ba and above) at $[\mathrm{Fe} / \mathrm{H}] \approx-3$. The VMSs produced no heavy $r$-elements but dominated chemical evolution at $[\mathrm{Fe} / \mathrm{H}]<-3$. This evolution resulted in a prompt inventory of "metals" corresponding to $[\mathrm{Fe} / \mathrm{H}] \approx-3$ in the IGM. Cessation of VMS activities and the rapid occurrence of a hypothesized SN II source for heavy $r$-elements then led to an explanation for the observed jump in the abundances of these elements at $[\mathrm{Fe} / \mathrm{H}] \approx-3$. The dominance of VMS activities at $[\mathrm{Fe} / \mathrm{H}]<$ -3 and the transition to normal astration at $[\mathrm{Fe} / \mathrm{H}] \approx-3$ are in accord with the theoretical study of Bromm et al. (2001). They showed that there is a critical metallicity corresponding to $-4<[\mathrm{Fe} / \mathrm{H}]<-3$ above which normal star formation should occur. Oh et al. (2001) showed that the number of VMSs required to produce $[\mathrm{Fe} / \mathrm{H}] \approx-3$ would also provide enough photons to reionize the IGM. Qian, Sargent, \& Wasserburg (2002) showed that the abundances of C, O, and Si in Ly $\alpha$ forests appear to be consistent with the corresponding prompt inventory. The observed lower bound of $[\mathrm{Fe} / \mathrm{H}] \approx-3$ for DLA systems was interpreted by Wasserburg \& Qian (2000b) as strong evidence in support of the prompt inventory model. Thus, there was a general accord between very different approaches.

There are now two considerations that require a reevaluation of the prompt inventory model. Qian \& Wasserburg (2003) proposed that the possible sources for heavy $r$-elements are accretion-induced collapse (AIC) of white dwarfs and low-mass $\left(\sim 8-10 M_{\odot}\right)$ SNe II, with the most likely source being AIC events. If AIC events instead of SNe II are the dominant source for heavy $r$-elements, the jump in the abundances of these elements at $[\mathrm{Fe} / \mathrm{H}] \approx-3$ can be attributed to the delay in turnon of these events relative to SNe II without invoking VMSs. We showed here that the growth of $[\mathrm{Fe} / \mathrm{H}]$ to -3 from a metalfree initial state is very rapid (in $\sim 30 \mathrm{Myr}$ ). So the probability of finding DLA systems with $[\mathrm{Fe} / \mathrm{H}]<-3$ is very low and the observed lower bound of $[\mathrm{Fe} / \mathrm{H}] \approx-3$ can no longer be considered as an argument in favor of the prompt inventory model. A prompt inventory corresponding to $[\mathrm{Fe} / \mathrm{H}] \approx-3$ is thus not required to explain the data on DLA systems but is also not in conflict with the interpretation of these data using the infall model. However, if there is no prompt inventory, a question remains regarding the approximately constant metallicity of the IGM over $1.5 \leq z \leq 5.5$ (Songaila 2001; Pettini et al. 2003). At present, it is not obvious how this can be explained by the infall model. Without VMSs associated with production of the prompt inventory, the source for reionization may also be a problem.

We wish to dedicate this Letter to Allan Sandage for galaxies of reasons. We thank Jason Prochaska for making available the new data that stimulated us to further consider DLA systems. Intense questioning by Michael Norman on the requirement of VMSs and a prompt inventory in our earlier models also provided a great stimulus. This work was supported in part by DOE grants DE-FG02-87ER40328, DE-FG02-00ER41149 (Y. Z. Q.), and DE-FG03-88ER13851 (G. J. W.), Caltech Division Contribution 8906(1108).

\section{REFERENCES}

Barkana, R., \& Loeb, A. 2001, Phys. Rep., 349, 125

Bromm, V., Ferrara, A., Coppi, P. S., \& Larson, R. B. 2001, MNRAS, 328, 969

Lu, L., Sargent, W. L. W., Barlow, T. A., Churchill, C. W., \& Vogt, S. 1996, ApJS, 107, 475

Oh, S. P., Nollett, K. M., Madau, P., \& Wasserburg, G. J. 2001, ApJ, 562, L1

Pettini, M., Madau, P., Bolte, M., Prochaska, J. X., Ellison, S. L., \& Fan, X. 2003, ApJ, 594, 695
Prochaska, J. X., Gawiser, E., Wolfe, A. M., Castro, S., \& Djorgovski, S. G. 2003, ApJ, 595, L9

Prochaska, J. X., \& Wolfe, A. M. 2000, ApJ, 533, L5 2002, ApJ, 566, 68

Qian, Y.-Z., Sargent, W. L. W., \& Wasserburg, G. J. 2002, ApJ, 569, L61

Qian, Y.-Z., \& Wasserburg, G. J. 2002, ApJ, 567, 515 2003, ApJ, 588, 1099

Songaila, A. 2001, ApJ, 561, L153

Wasserburg, G. J., \& Qian, Y.-Z. 2000a, ApJ, 529, L21 . 2000b, ApJ, 538, L99 